\title{
A stream of cells migrating from the caudal telencephalon reveals a link between the amygdala and neocortex
}

\author{
Ryan Remedios $^{1}$, Dhananjay Huilgol ${ }^{1}$, Bhaskar Saha ${ }^{1}$, Padmanabhan Hari ${ }^{1}$, Lahar Bhatnagar ${ }^{1}$, \\ Thomas Kowalczyk ${ }^{2}$, Robert F Hevner ${ }^{2-4}$, Yoko $\mathrm{Suda}^{5}$, Shinichi Aizawa ${ }^{5}$, Toshio Ohshima ${ }^{6,7}$, \\ Anastassia Stoykova ${ }^{8}$ \& Shubha Tole ${ }^{1}$
}

The amygdaloid complex consists of diverse nuclei that belong to distinct functional systems, yet many issues about its development are poorly understood. Here, we identify a stream of migrating cells that form specific amygdaloid nuclei in mice. In utero electroporation showed that this caudal amygdaloid stream (CAS) originated in a unique domain at the caudal telencephalic pole that is contiguous with the dorsal pallium, which was previously thought to generate only neocortical cells. The CAS and the neocortex share mechanisms for specification (transcription factors Tbr1, Lhx2 and Emx1/2) and migration (reelin and Cdk5). Reelin, a critical cue for migration in the neocortex, and Cdk5, which is specifically required for migration along radial glia in the neocortex, were both selectively required for the normal migration of the CAS, but not for that of other amygdaloid nuclei. This is first evidence of a dorsal pallial contribution to the amygdala, demonstrating a developmental and mechanistic link between the amygdala and the neocortex.

The role of the amygdala as a key mediator of behavior has motivated great interest in understanding its complexity. There is, however, a dearth of information about how this complexity arises in development. The neuroepithelial origins of the cells that comprise many of the different amygdaloid nuclei, as well as the mechanisms that control their migration, are not well understood.

Current models of amygdaloid complex development identify specific domains near the pallial-subpallial boundary (PSB) as the source for several components of the amygdala. These domains reside at the interface of dorsal and ventral structures in the telencephalon. This adds a level of complexity in that the amygdala is composed of dorsal (pallial) and ventral (subpallial) derivatives ${ }^{1,2}$. The basolateral amygdaloid complex shows pallial characteristics and is believed to originate from specific domains near the PSB called the lateral and ventral pallium ${ }^{1,3,4}$. Ventrally migrating streams from the lateral and ventral pallium populate these amygdaloid nuclei. Transcription factors Pax6 and Tlx, both regulators of the ventral and lateral pallium domain boundaries, also control the development of these nuclei ${ }^{5,6}$.

Little is known about the development of the cortical amygdaloid nuclei, however. These nuclei are considered to be 'cortical' in nature, as they show a layered morphology and are considered to be trilaminar extensions of the piriform cortex ${ }^{7,8}$. They are part of the 'olfactory amygdala', as they receive direct olfactory input from the main olfactory bulb as well as the accessory olfactory bulb ${ }^{9,10}$. However, many developmental issues remain to be clarified with respect to these structures. In particular, these nuclei express the full complement of 'pallial' markers, similar to nuclei of the basolateral complex ${ }^{1,4}$. Although their pallial identity is unquestionable, it is unclear which pallial domain they arise from, with the ventral pallium being proposed as the likely source ${ }^{1,11}$.

The nucleus of the lateral olfactory tract (nLOT) is of particular interest. This structure is connected with the main olfactory bulb and the piriform cortex and is implicated in nonpheromonal behavior such as feeding ${ }^{10,12}$. It has been described as a three-layered structure; however, in two recent studies, we showed that layer 2 of this nucleus (nLOT2) appears to require developmental transcription factors that do not appear to be necessary for layer 1 or for surrounding amygdaloid structures ${ }^{6,13}$. Here, we report a finding that sheds some light on this unusual aspect of the nLOT2. We found that this structure, one of the most rostral among the amygdaloid cell groups, originates at the caudal extreme of the telencephalic neuroepithelium. The origin appears to reside in the dorsal pallium, which was thought to exclusively generate the neocortex. From this caudal origin, a migrating stream of cells winds a path rostrally, terminating in the nLOT2.

\footnotetext{
${ }^{1}$ Department of Biological Sciences, B-304, Tata Institute of Fundamental Research, Homi Bhabha Road, Colaba, Mumbai 400005, India. ${ }^{2}$ Department of Pathology, ${ }^{3}$ Center on Human Development and Disability, ${ }^{4}$ Institute for Stem Cell and Regenerative Medicine, University of Washington, Seattle, Washington 98195-7270, USA. ${ }^{5}$ Laboratory for Vertebrate Body Plan, Center for Developmental Biology, 2-2-3 Minatojima-minamimachi, Chuo-ku, RIKEN, Kobe 650-0047, Japan. 6 Laboratory for Developmental Neurobiology, Brain Science Institute, RIKEN, Saitama 351-0198, Japan. 7 Department of Life Science and Medical Bio-Science, Waseda University, 3-4-1 Okubo Shinjuku-ku, Tokyo 169-8555, Japan. ${ }^{8}$ Max-Planck Institute of Biophysical Chemistry, Am Fassberg 11, D-37077 Göttingen, Germany. Correspondence should be addressed to S.T. (stole@tifr.res.in).
} 
a
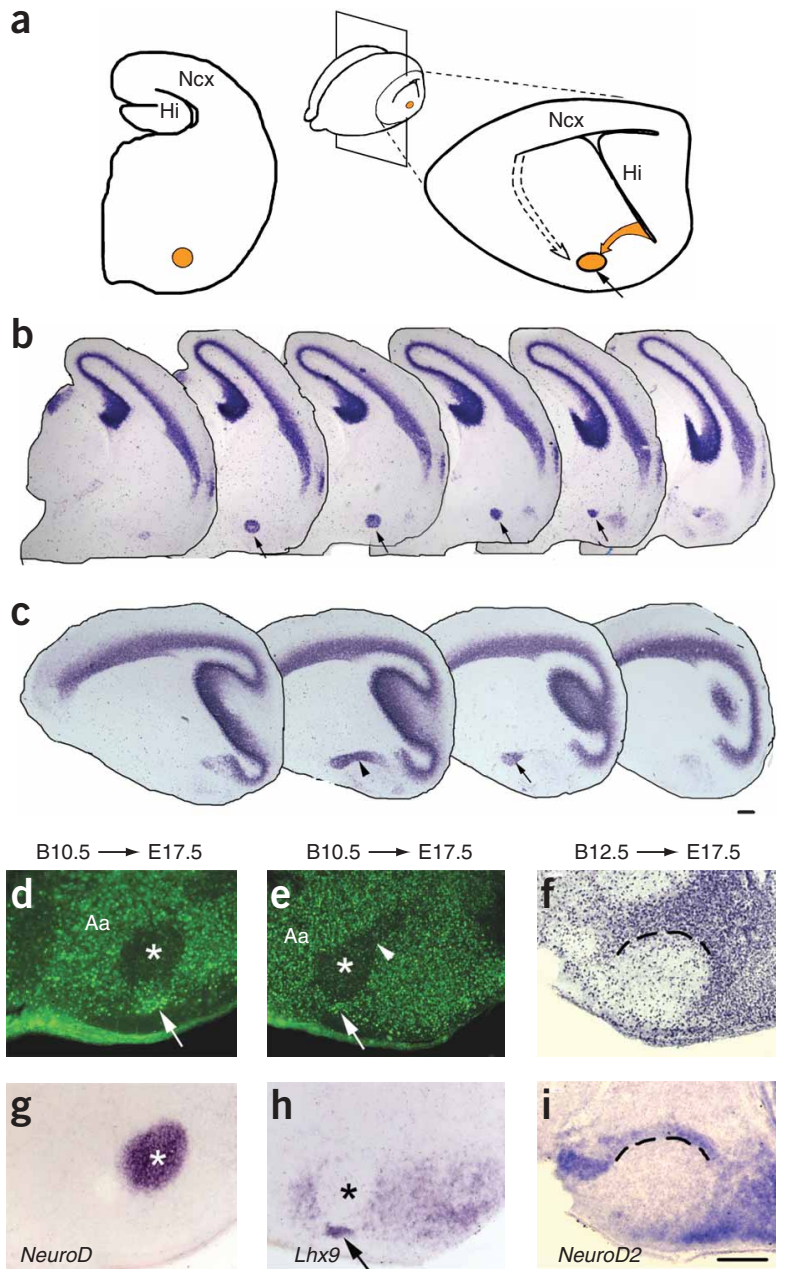

Exploring the mechanisms that regulate this migration revealed further disparities between the nLOT2 and the rest of the amygdaloid complex. These included a requirement for both reelin and $\mathrm{Cdk} 5$, a molecule critical for radial glial migration in the neocortex, for proper nLOT2 migration. The origin of the nLOT2 in the dorsal pallium presents the first developmental connection between the amygdala and the neocortex and raises the possibility of a shared evolutionary history between these structures.
Figure 1 A stream of amygdaloid cells from the caudal telencephalon. (a) Schematics showing the coronal plane of sectioning (rectangle) and the sagittal plane (dashed lines). (b) A series of coronal sections at E15.5 showed the nLOT2 as a globular structure in the ventrolateral region of the brain, marked by NeuroD expression (yellow circles, a; arrows, a,b), appearing at multiple levels of sectioning. (c) The contralateral hemisphere of the same brain, cut in the sagittal plane, revealed a NeuroD-expressing arc of labeling (arrowhead) extending from the caudal telencephalic neuroepithelium, and terminating in the nLOT2 (arrow). The dashed arrow in a indicates the standard migration path of amygdaloid precursors, which is orthogonal to the stream that terminates in the nLOT2. (d) A BrdU pulse administered at E10.5 (B10.5) labeled cells in the nLOT1 (arrow) and anterior amygdaloid areas by E17.5, but left the nLOT2 poorly labeled (asterisk, d). (e) This is also seen in sagittal sections of littermate embryos (asterisk), in which the path of the CAS is also poorly labeled (arrowhead). (f) A BrdU pulse administered at E12.5 (B12.5) labeled cells in the arc. (g-i) The nLOT2 was identified by comparing with corresponding serial sections showing NeuroD expression (asterisk, $\mathbf{g}$ ), and by exclusion of $L h \times 9$ expression (asterisk, $\mathbf{h}$ ). The nLOT1 was identified by $L h x 9$ expression (arrow, h). The arc was identified by NeuroD2 expression in serial sections (dashed lines $f, i$ ). Aa, anterior amygdala; $\mathrm{Hi}$, hippocampus; Ncx, neocortex. Orientation in all sagittal sections: rostral is left, caudal is right. Scale bars are $200 \mu \mathrm{m}$.

\section{RESULTS}

\section{A stream from the caudal telencephalon}

The nLOT2 is known to express several pallial markers and is seen as a typical cup-shaped structure in coronal sections ${ }^{1,4,6,13}$. NeuroD1 (Neurogenic differentiation 1; referred to as NeuroD) expression identified this nucleus in a series of coronal sections at embryonic day (E) 15.5 (Fig. 1a,b). Because the nLOT2 appeared to extend through several sections, we sought to examine this nucleus in the sagittal plane. We observed that NeuroD expression delineated a curved arc, extending from the caudal telencephalic neuroepithelium up to the nLOT2 (Fig. 1a,c). In horizontal sections, the rounded nLOT2 appeared to be connected with the caudal neuroepithelium by a distinct row of NeuroD-expressing cells (Supplementary Fig. 1 online). This suggested that there was a stream of cells migrating from the caudal neuroepithelium, the CAS, and terminating in the nLOT2 and provided the first hint of a previously unknown source for this amygdaloid nucleus.

Notably, layer 1 of the nLOT (nLOT1) did not appear to arise from the CAS, as it was not identified by any of the markers that are expressed in the arc or in the nLOT2. BrdU birth-dating studies provided further evidence to support the idea that the nLOT1 and 2 have distinct developmental origins. The nLOT1 is densely populated by cells that were labeled with a BrdU pulse at E10.5, but there was no labeling in the nLOT2 or in the CAS (Fig. 1d,e). In contrast, BrdU
Figure 2 In utero electroporation identifies a unique caudal origin for the CAS.

(a-d) In utero electroporation of an enhanced GFP-expressing construct at E11.5, restricted to a small portion of the caudal telencephalic neuroepithelium, revealed a stream of GFP-expressing cells at E15.5 (arrow, b). A serial section showing NeuroD expression indicated that this stream was the CAS (arrow, a). The domain of electroporation in the ventricular zone was also marked by GFP expression, and was seen to include the caudal extreme of the telencephalon (arrowheads, $\mathbf{b}-\mathbf{d}$ ). Notably, sections through the entire brain revealed that no other portion of the

ventricular zone was marked by GFP expression, indicating that the labeled cells must have arisen from some portion of the caudally electroporated source. (e-g) High magnification views of the CAS in $\mathbf{a}(\mathbf{e})$ and $\mathbf{b}(\mathbf{f})$ and a false-color overlay, $\mathbf{g}$. Orientation: rostral is left, caudal is right. Scale bars are $200 \mu \mathrm{m}$.
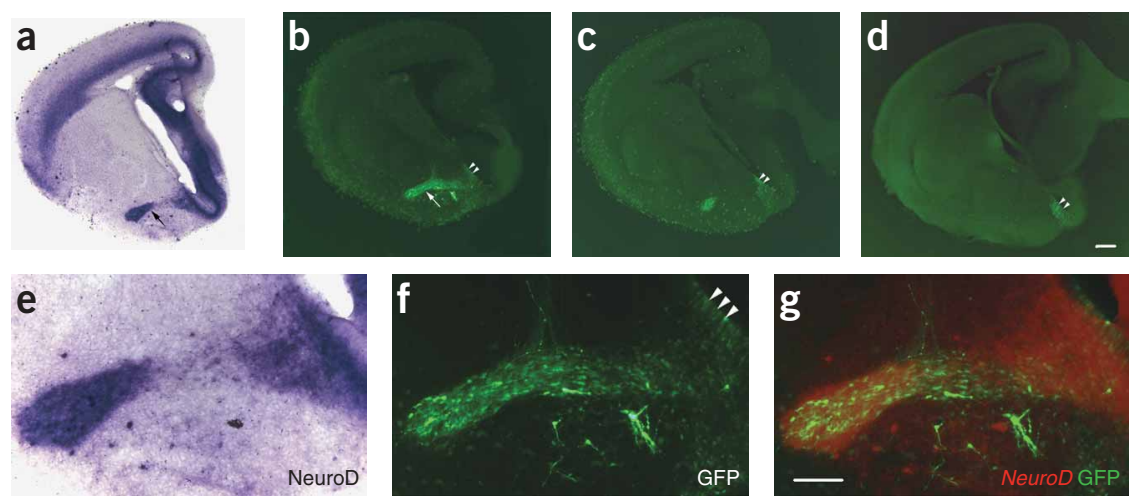

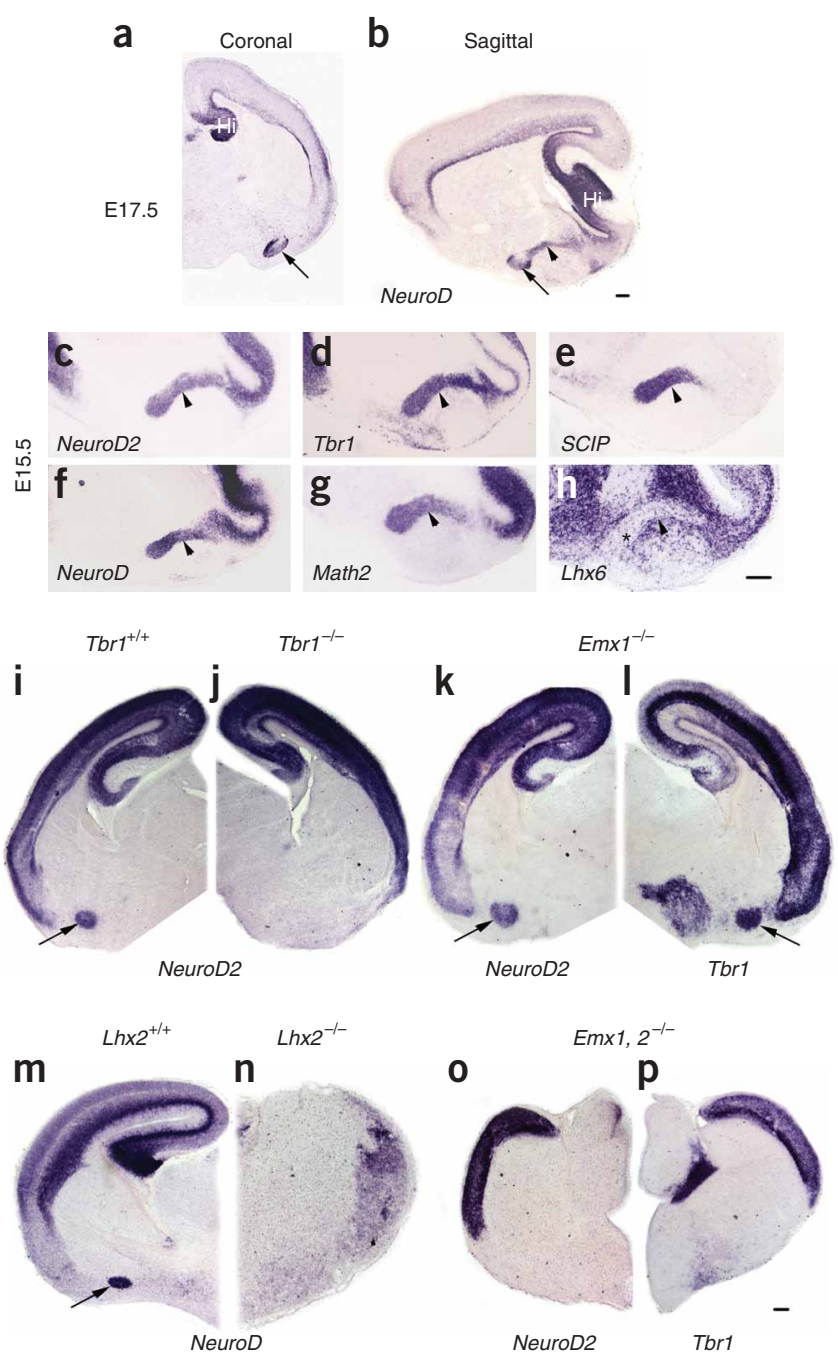

pulses given on E11.5 and E12.5 identified cells both in the CAS and the nLOT2, which was consistent with previous reports ${ }^{14}$ (Fig. 1f).

To establish whether the arc-like labeling of NeuroD indeed identifies a migrating stream of cells, we used in utero electroporation to introduce a green fluorescent protein (GFP)-expressing construct into a small portion of the caudal neuroepithelium at E11.5. When we examined it $4 \mathrm{~d}$ later, at E15.5, we saw that the labeled neuroepithelium gave rise to a stream of cells that extended rostrally in an arc-like trajectory that overlapped well with NeuroD expression (Fig. 2). Because we introduced the GFP-expressing construct into the ventricles, electroporation was only able to transfect cells of the ventricular zone. GFP-expressing cells in the mantle layers must arise from some portion of the labeled ventricular zone. The domain of electroporation in the ventricular zone continued to express GFP for several days and was therefore easily identified. Examination of the entire brain indicated that only a limited portion of the caudal neuroepithelium was electroporated (arrowheads, Fig. 2b-d). Therefore, this region of the caudal telencephalic neuroepithelium must contain the origin of a stream of cells that extends rostrally up to and including the nLOT2.

Multiple unrelated markers labeled the CAS as early as E15.5 (Fig. 3). These included well-known pallial markers of the bHLH family: NeuroD, NeuroD2, NeuroD6 (referred to as Math2), Neurogenin2 (Neurog2, data not shown), POU-domain gene SCIP and T-box brain gene 1, Tbr1. Tbr1 was expressed in glutamatergic neurons in
Figure 3 Multiple pallial markers identify the CAS and the nLOT2 emerging from the caudal telencephalic neuroepithelium. (a,b) Coronal (a) and sagittal sections (b) showing NeuroD expression at E17.5 revealed a mature, cupshaped nLOT2 (arrow). (c-g) In E15.5 sagittal sections, the expression of several pallial markers identified the CAS as an arc of labeled cells (arrowheads) from the caudal neuroepithelium extending up to the nLOT2. (h) This stream was specifically excluded by subpallial marker $L h x 6$. (i-n) The $\mathrm{nLOT} 2$ was detectable in coronal sections of control brains, but not in sections of $\operatorname{Tbr} 1(\mathbf{i}, \mathbf{j})$ or $\operatorname{Lh} \times 2(\mathbf{m}, \mathbf{n})$ mutant brains. Emx1 (single) mutants had a well-defined nLOT2 expressing both NeuroD2 (k) and Tbr1 (I). (o,p) Emx1/2 double mutants did not show this structure with either marker. Orientation in all sagittal sections: rostral is left, caudal is right. Scale bars are $200 \mu \mathrm{m}$.

the pallium; consistent with this, markers of the subpallium such as Lhx6 (Fig. 3) and interneuron marker Glutamic acid decarboxylase 1 (Gad1, data not shown) selectively excluded the CAS.

By E17.5, the CAS had given rise to its derivatives; the cup-like nLOT2 was well formed, and other cells in the CAS appeared to coalesce into the amygdaloid components known as intercalated cell masses (ICM; arrowhead, Fig. 3b). This is consistent with a previous study ${ }^{4}$, which reports a mixed cellular constitution for the ICM, arising from pallial and subpallial domains, and suggests that the pallial component of the ICM may in fact be a 'caudal extension of the nLOT2'. Our present findings offer a means by which these two contiguous structures arise in development from a common stream of migrating cells, the CAS.

Transcription factors that are expressed in early stages of pallial development, either in the neuroepithelium (LIM homeobox protein 2, Lhx2, and empty spiracles homolog 1 and 2, Emx1 and Emx2) or in the postmitotic neurons (Tbr1), were also required for the development of the nLOT2. This nucleus was not detectable in either Tbr1 mutant or Emx1 Emx2 double-mutant mice but was present in the Emx1 and Emx2 (single) mutant mice ${ }^{6}$ (Fig. 3). The requirement for Tbr1 and both Emx1 and Emx2 is particularly significant, as these transcription factors are also required for the specification of selective cohorts of early-born neurons in the cortex ${ }^{15,16}$. In the $L h x 2$ mutant, the nLOT2 was selectively missing, whereas other amygdaloid nuclei were present ${ }^{13}$ (Fig. 3). The neocortex is also missing in the $L h \times 2$ mutant ${ }^{17}$, suggesting a potentially similar requirement for Lhx2 in CAS and the cortex.

\section{The CAS originates in the dorsal pallium}

The site of origin of the CAS in the ventricular zone of the telencephalic neuroepithelium needed careful analysis, as the caudal telencephalon has not thus far been analyzed in detail with respect to neuroepithelial domains at the PSB. The lateral and ventral pallium are currently thought to be the sites where specific amygdaloid nuclei arise ${ }^{1,3,4}$. In particular, the olfactory amygdala is thought to be part of the ventral pallium $^{1,11}$. We therefore examined the ventral pallium in the caudal telencephalic neuroepithelium. At E14.5, the earliest stage at which the CAS was detectable, Tbr1 labeled postmitotic cells in the entire pallium. In the caudal telencephalon, this labeling extends into the CAS and the nLOT2 (Figs. 3d and 4). To our surprise, an adjacent section probed for the ventral pallium marker secreted frizzled-related protein $2(s F r p 2)^{18}$ indicated that the CAS appeared to arise from a ventricular zone domain that was ventral to the ventral pallium, instead of from the ventral pallium itself (Fig. $\mathbf{4 b}, \mathbf{e}$ ). We probed the molecular nature of this ventral domain and found that it expressed the well-defined pallial markers Emx1 and NeuroD2 (Fig. 4g-1). Emx1 expression was particularly notable, as this gene has been described to exclude the ventral pallium ${ }^{1,3,4}$. NeuroD2 expression overlapped partially with that of the sFrp2 domain in the ventricular zone, but the stream appeared to originate from a more ventral domain. This was reinforced by 
examining Lhx6, a marker of subpallial interneurons, which was present in a 'corridor' of cells lining the trajectory of the CAS (Fig. 3h; arrowheads, Fig. 4m). This corridor appeared to begin from an extremely ventral position in the neuroepithelium (asterisk, Fig. 4o), clearly excluding the domain of $s F r p 2$ expression.

Genetic evidence supports these gene expression data, as the Emx1/2 double-mutant mice (but not either single mutant) lacked the nLOT2 (Fig. 3); the origin of this structure must lie in the region of neuroepithelium that expresses both genes. Emx2 has a wider expression than Emxl, which excludes the ventral pallium. Together, the data indicate that the CAS does not originate in the ventral pallium ventricular zone but in an Emxl-expressing domain that is just adjacent and ventral to the ventral pallium.

The question then arises as to which component of the pallium the CAS origin domain belongs to. In much of the telencephalon, the subpallium is present ventral to the ventral pallium. At extreme caudal levels, however, the subpallium is not present, so the ventral pallium would be expected to be contiguous with the medial pallium. Indeed,
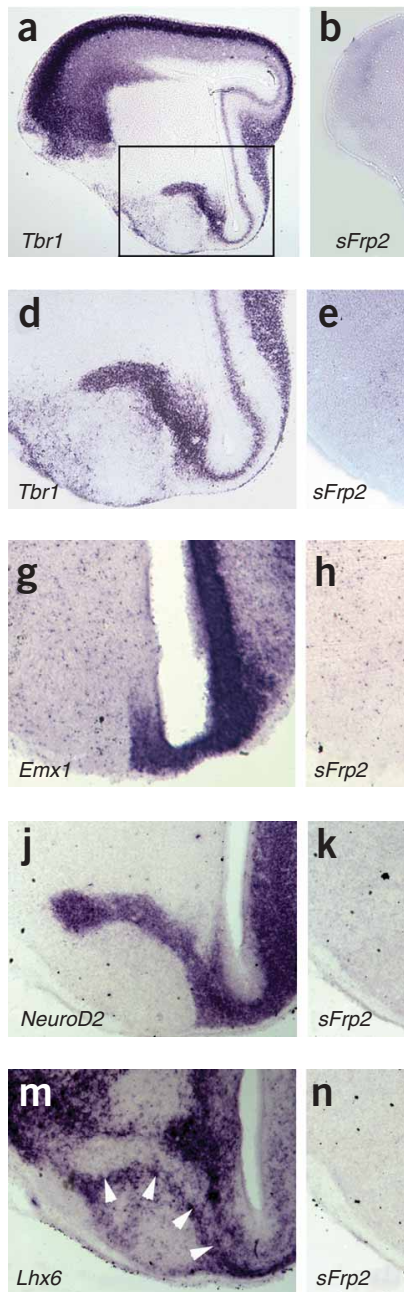
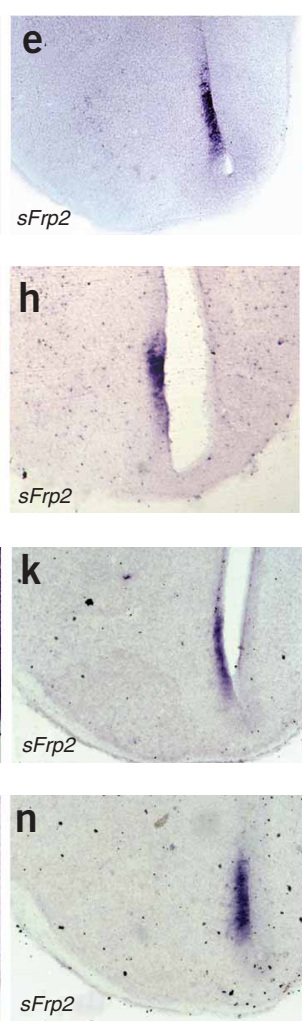
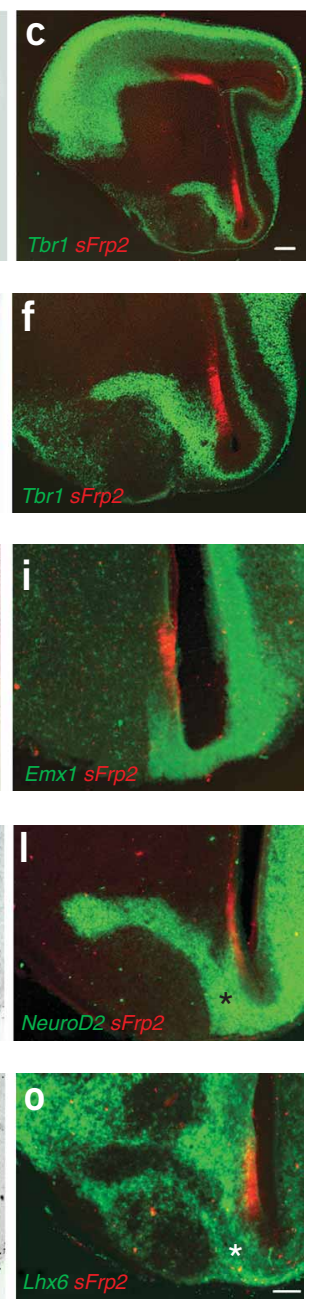
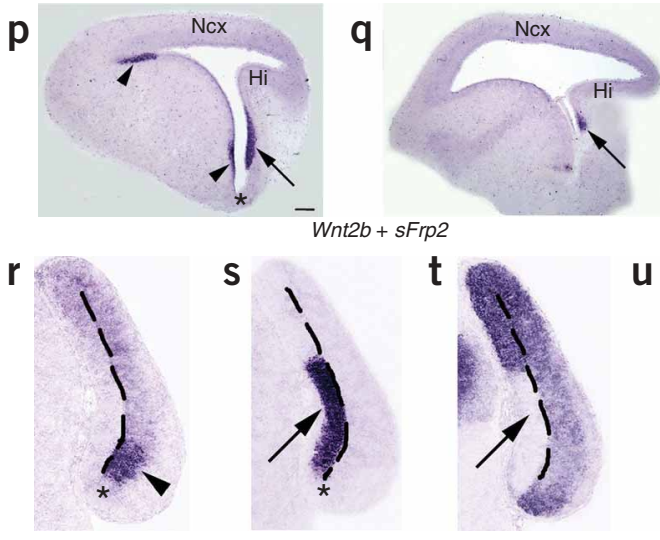

$\mathbf{S}$
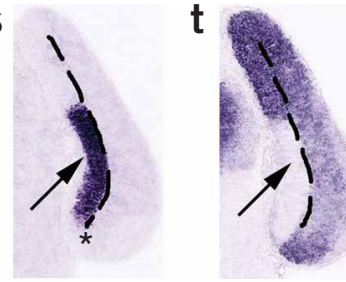

$\mathbf{u}$

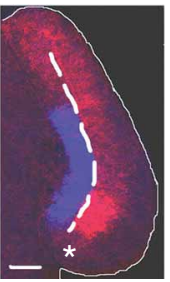

Lhx2

sFrp2Wnt2b

$\mathbf{v}$

Whit2b
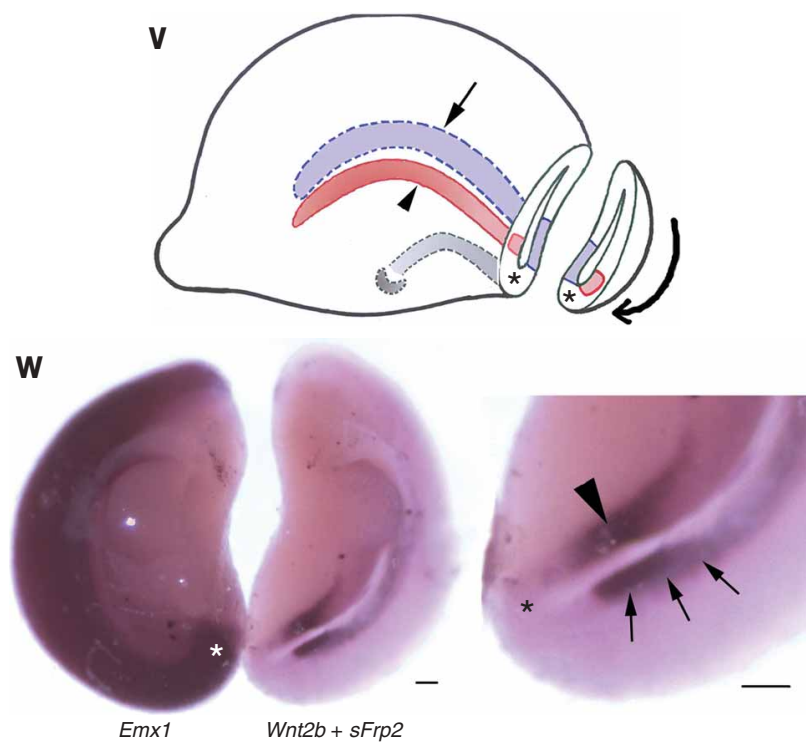

Figure 4 The CAS originates in the dorsal pallium. (a-c)Tbr 1 expression was seen in the entire pallium, including the neocortex and hippocampus, and also in the CAS. (b) sFrp2 expression marked the ventral pallium/antihem. (d-f,j-I)Higher magnification views of the CAS in sections expressing either Tbr 1 or NeuroD2 suggested that the CAS originated in a domain adjacent and ventral to the $s F r p 2$-positive ventral pallium. (g-i) Emx1 expression excluded the ventral pallium and marks the neuroepithelium ventral to the sFrp2 domain. (m-0) Lhx6 expression identified a corridor of interneurons flanking the trajectory of the CAS, which originated well outside of the sFrp2 domain of expression (asterisk, o). (p, q) Combined expression of hem marker Wnt2b (arrow) and ventral pallium marker sFrp2 (arrowheads) showed that both structures are present at sectioning levels that also showed the CAS (compare $\mathbf{p}$ with $\mathbf{a}, \mathbf{b})$. In more medial sections (q), only the hem was seen, adjacent to the hippocampus. The hem, however, was some distance away from the CAS origin, and there was an intervening domain between the hem and the ventral pallium that expressed neither marker (asterisk, $\mathbf{p}$ ). $(\mathbf{r}-\mathbf{u})$ This domain was also seen in coronal sections at E12.5, which revealed the sFrp2-expressing ventral pallium (arrowhead, $\mathbf{r}$ ) and the Wnt2b-expressing hem (arrow, s). An intervening neuroepithelial domain, corresponding to the CAS origin, did not express either marker, and showed high $L h x 2$ expression (t, compare with asterisk in $\mathbf{r}, \mathbf{s}, \mathbf{u})$. (v) A schematic of an E12.5 hemisphere viewed from the lateral aspect showed the ventral pallium (arrowhead, red domain) and the hem (arrow, blue domain) extending along the lateral and medial faces of the telencephalon, respectively. These domains did not meet, but were separated by a portion of the dorsal pallium that extended around the caudal pole of the telencephalon (curved arrow) and appeared ventrally (asterisk). (w) E12.5 whole mount preparations viewed from the ventromedial aspect showed Emx1 expression (white asterisk, w). There was a distinct gap between the hem (arrows) and the ventral pallium (arrowhead). Only the most caudal end of the ventral pallium was seen in the whole mount (arrowhead, $\mathbf{w}$ ), as the rest of this domain extended along the lateral side of the hemisphere, and was hidden in this view by the ganglionic eminences. Orientation, in all sagittal sections: rostral is left, caudal is right. Scale bars are $400 \mu \mathrm{m}$ (a-c,p,q) and $200 \mu \mathrm{m}$ for all other panels. Hi, hippocampus; Ncx, neocortex. 

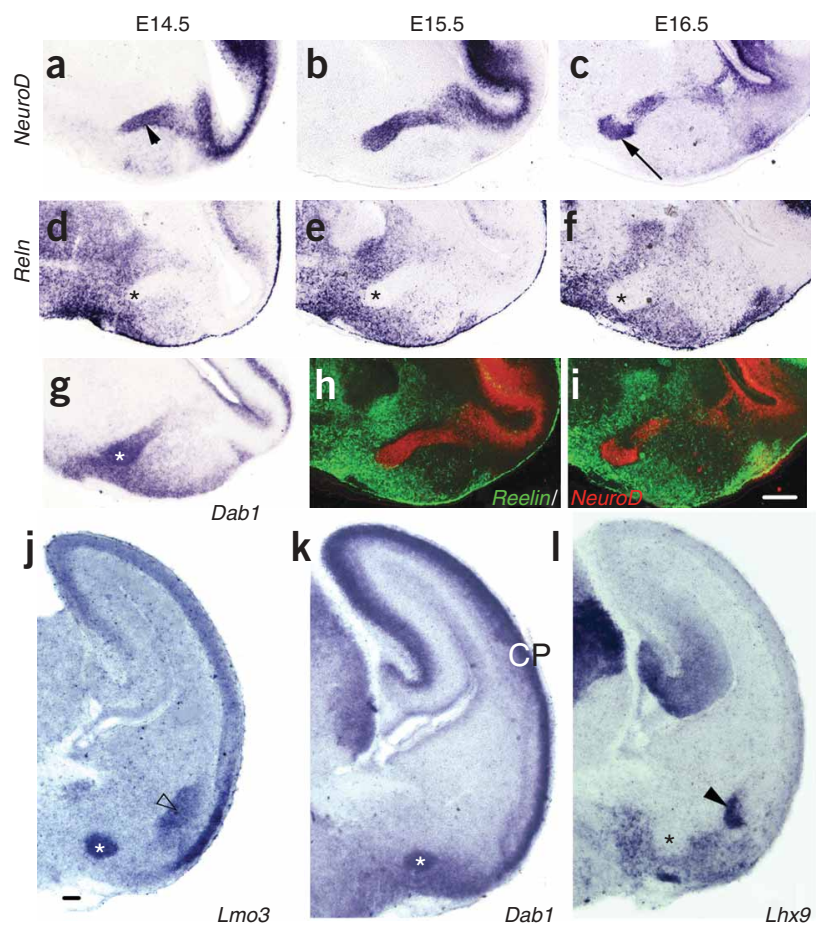

the telencephalic signaling center, the cortical hem, which is the most medial component of medial pallium, was thought to join with the ventral pallium (antihem) at the caudal end of the telencephalon. This interpretation was made on the basis of lateral or medial views of intact telencephalic hemispheres processed for hem marker Wingless related MMTV integration site $2 b$ (Wnt2b) and antihem marker sFrp2 (ref. 19). However, when examined in sections at E14.5 (Fig. 4p,q) and
Figure 5 The CAS migrates in a reelin-free corridor. (a-c) NeuroD expression at E14.5 (a), E15.5 (b) and E16.5 (c) showed an arc of labeling that progressed with developmental age, beginning as a short spur of labeling at E14.5 (arrowhead, a), and extending rostrally, to terminate in the cup-shaped $\mathrm{nLOT} 2$ by E16.5 (arrow, c). (d-f) Serial sections probed for Reln expression at each stage indicated that the path of the CAS appeared devoid of labeling, whereas surrounding regions showed strong expression. (h,i) False-color overlays of $\mathbf{b}$ and $\mathbf{e}$, and $\mathbf{c}$ and $\mathbf{f}$, respectively. Asterisk (d-f) indicates the rostral most point of the stream. (g) An E15.5 sagittal section revealed strong $D a b 1$ expression in the $\mathrm{nLOT} 2$ and anterior amygdala, and weaker expression in the CAS. (j-l) Serial coronal sections at E15.5 comparing $L m o 3$ (j), $L h \times 9$ (k) and Dab1 (I) expression indicate that the lateral amygdala/basolateral nucleus (open arrowhead, $\mathbf{j}$ ), and basomedial nucleus (arrowhead, I) did not express Dab1. In contrast, the nLOT2 (asterisk, $\mathbf{j , k}, \mathbf{k}$ ) and the cortical plate (CP) showed robust expression. Orientation in all sagittal sections: rostral is left, caudal is right. Scale bars are $200 \mu \mathrm{m}$.

E12.5 (Fig. 4 r-u), both stages clearly revealed a 'gap' between the ventral pallium (antihem) and the medial pallium (hem) (asterisk, Fig. $4 \mathbf{p}-\mathbf{u})$. This region expressed high levels of $L h x 2$, a marker which was notably absent from the hem (Fig. 4t), but was expressed at high levels in the dorsal pallium and at markedly lower levels in the ventral pallium and subpallium ${ }^{6,17}$.

It appears, therefore, that a portion of the pallium that is neither the ventral pallium nor the hem extends around the caudal pole of the hemisphere and was seen ventrally, protruding in between these two structures (curved arrow, Fig. 4v). This interpretation is reinforced by examining intact telencephalic hemispheres that are turned over to present a ventral view (Fig. 4w). A distinct gap appeared between Wnt2b and sFrp2 expression at the extreme caudal pole of the telencephalon. Emxl expression, in contrast, curved around the caudal pole (asterisk, Fig. 4w), indicating that the neuroepithelium of the gap is an Emx1-positive component of the pallium. This domain is contiguous with the dorsal pallium. Because it expresses Emxl, as well as a broad array of dorsal pallium markers, we conclude that the
Figure 6 The CAS migration is abnormal in the reeler mutant. (a-d) At E15.5, NeuroD and NeuroD2 expression identified the CAS in sagittal sections of control brains $(\mathbf{a}, \mathbf{c})$. In reeler mutants (b,d) a shorter stream was observed (compare dashed and dotted lines in $\mathbf{c}, \mathbf{d}$ ), which showed an aberrant trajectory (compare arrowheads in $\mathbf{a}-\mathbf{d}$ ). (e-h) At PO, Tbr1 (e) and NeuroD2 (g) identified the mature $\mathrm{nLOT} 2$ in sagittal sections of control brains (arrows), being weakly expressed in this structure, but barely detectable in the path of the stream. Reeler mutant brains $(\mathbf{f}, \mathbf{h})$ showed higher expression of these genes in a globular nLOT2like structure (arrows), which continued to occupy a position much closer to the ventricular zone than it did in control brains (compare dashed and dotted lines in $\mathbf{g , h}$ ). Unlike the control, the mutant CAS also maintained strong labeling in the stream at this stage (arrowheads, $\mathbf{f}, \mathbf{h}$ ). Orientation: rostral is left, caudal is right. (i) In coronal sections of control brains at E15.5, Lmo3 expression identified the nLOT2 (asterisk), as well as the globus pallidus and lateral amygdala at mid-levels of sectioning. (j,k) A false-color overlay of serial sections (k) showed $L h \times 9$ expression (j) in a complementary pattern, excluding the nLOT2 (asterisk, j), but labeling surrounding anterior amygdaloid structures, and also identifying the nLOT1 (arrow, j). (I-n) Reeler mutant brains did not show an Lmo3-positive nLOT2 at comparable levels, although the globus pallidus and lateral amygdala were observed (I). Serial sections probed for $L h \times 9$ also showed normal labeling in surrounding anterior amygdaloid areas, as well as in the nLOT1 (arrow, $\mathbf{m}$ ), but did not exclude a 'cavity' for the nLOT2. (o-q) At caudal levels in the reeler mutant, a globular Lmo3-positive structure was seen to nest in an $L h \times 9$-negative cavity (asterisk, $\mathbf{p}$; q), consistent with the ectopic location of the nLOT2-like structure in the mutant. G, globus pallidus; L, lateral amygdala. Scale bars are $200 \mu \mathrm{m}$. 

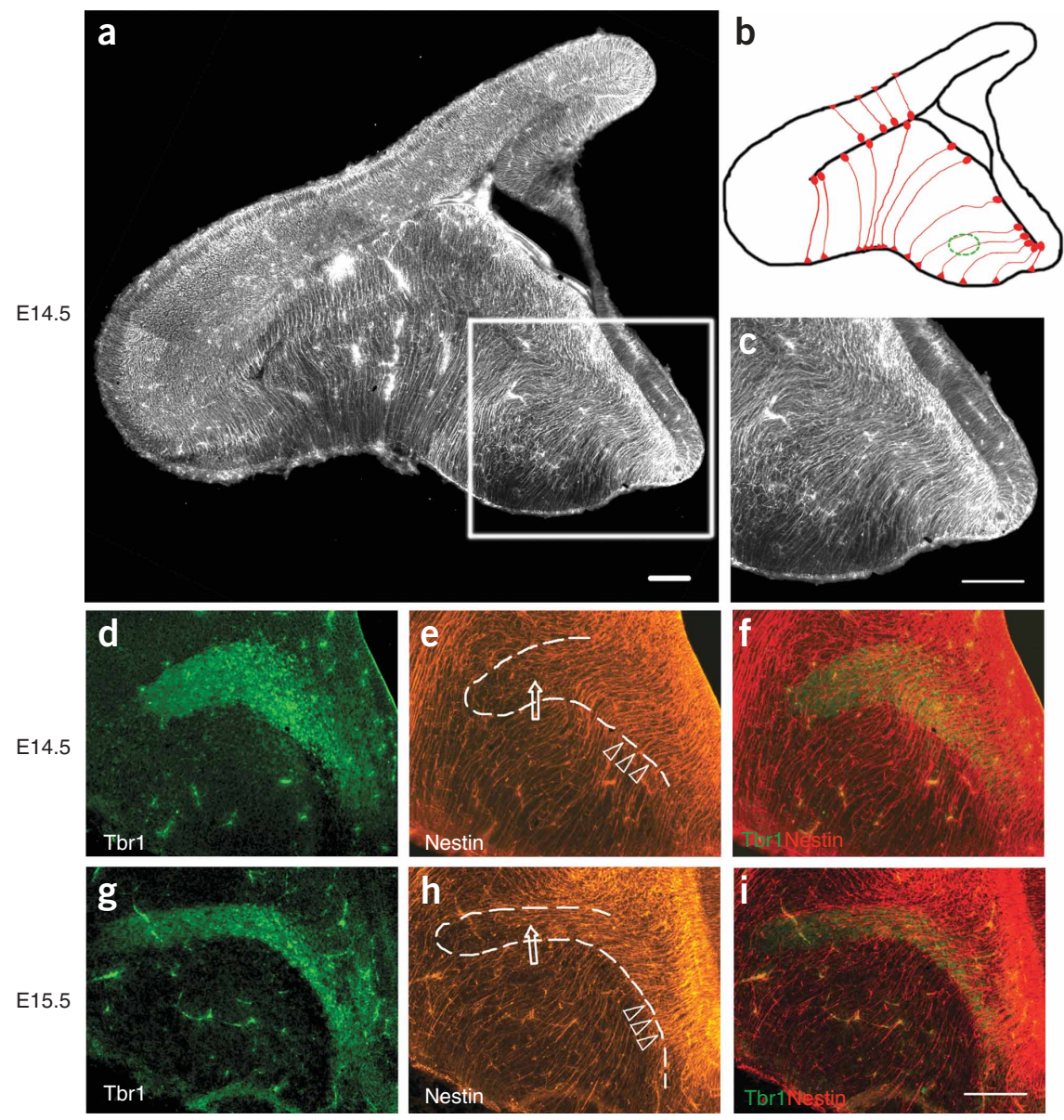

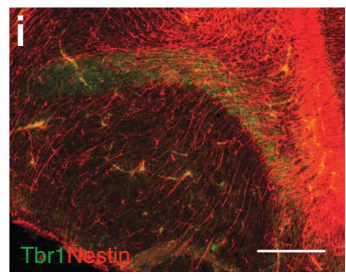

Figure 7 Radial glia and the CAS trajectory. (a,b) A montage of 32 individual frames

(a) revealed Nestin-positive radial glial orientation as seen in sagittal sections at E15.5, schematized in (b). The dashed green circle marks the approximate location of the nLOT2. (c) High magnification of the radial glial palisade at the caudal pole of the telencephalon. (d-i) Tbr 1 and Nestin double immunostaining at E14.5 (d-f) and E15.5 (g-i). At both ages, the CAS trajectory (dashed lines) was initially orthogonal to the radial glial palisade (arrowheads), and then turned to align itself with the radial glia (arrows). Orientation: rostral is left, caudal is right. Scale bars are $200 \mu \mathrm{m}$.

brains (Fig. 6; compare dashed lines and dotted lines in control and mutant sections). Postnatally, the mutant nLOT2-like structure continued to occupy an aberrant position (compare dashed and dotted lines in control and mutant sections), appeared to be spherical and failed to assume the cup-like morphology that was achieved in normal embryos several days earlier. Furthermore, the migrating cells appeared to be trapped in the path of the stream and did not coalesce into ICM-like structures. Additionally, at postnatal stages, the reeler mutant CAS continued to express higher levels of Tbr1, Lmo3 and NeuroD2, resembling the expression at embryonic stages in control brains (Fig. 6). Together, these results indicate that in the absence of reelin, the CAS derivatives appear immature and also

CAS arises from the dorsal pallium, a domain that has not previously known to contribute to the amygdala.

\section{The CAS requires reelin for proper migration}

The migration path of the CAS motivated us to carry out a stage-wise examination of its development. The CAS appeared as distinct 'spur' emerging from the caudal telencephalic neuroepithelium at E14.5. Subsequently, the tip of the CAS became globular, with the characteristic cup-like shape of the nLOT2 being apparent by E16.5 (Fig. 5a-c). Notably, we found that serial sections at each stage probed for Reln (reelin) expression revealed that the path of the CAS itself was devoid of labeling; as the CAS moved forward, it appeared to create a reelin-free corridor in territory that is rich in Reln expression (Fig. 5d-f). Furthermore, Disabled homolog 1 (Dab1), a key cytosolic component of the reelin signaling pathway, was expressed in the newly formed nLOT2, the CAS, and in the cortical plate (Fig. 5g-1). Notably, no detectable Dab1 expression was seen in the nuclei of the basolateral complex, which are pallial derivatives ${ }^{4}$. These structures were identified in serial sections by the expression of LIM domain only 3 (Lmo3) in the lateral amygdala and basolateral amygdala and $L h \times 9$ expression in the basomedial nucleus (ref. 13; Fig. 5j-1). Therefore, as in the cortex, a reelin-dependent mechanism could serve to guide the migration of the CAS and its derivatives.

To test this hypothesis, we examined brains from reeler mutant embryos. Reeler brains showed an aberrant CAS at embryonic and postnatal stages (Fig. 6). At both stages, although the stream appeared to terminate in a globular nLOT2-like structure, it was mislocated dorsally and was much closer to its neuroepithelial origin than it was in control have defective morphology and positioning, indicating that reelin is critical to the development of these structures.

\section{The nLOT1 and other nuclei are normal in the reeler mutant}

In marked contrast, the nLOT1, which is not a CAS derivative, did not appear to require reelin for its migration. In control brains, coronal sections revealed that the nLOT1 and nLOT2 were at the same level of sectioning, as indicated by $L h x 9$ and $L m o 3$ expression, respectively. At similar levels in the reeler mutant, level-matched by the presence of the globus pallidus and the lateral amygdala, the nLOT1 was present and marked by Lhx9 expression. It is only the nLOT2 that was missing from this level and instead appeared only at more caudal levels (Fig. 6l-q). It is only the positioning, not the specification, of the nLOT2 that was perturbed, as it expressed $L m o 3$ and excluded $L h x 9$, as was the case in control brains (Fig. 5). Other amygdaloid structures, in particular other components of the pallial amygdala, appeared to be normal; the anterior amygdala, lateral amygdala, basolateral amygdala and basomedial nucleus were all identified at appropriate levels (Fig. 61-q), indicating that only the CAS derivatives are dependent on reelinguided mechanisms for their migration.

\section{A glial palisade aligns with part of the CAS trajectory}

Migration along radial glia constitutes a major cellular mechanism for cell movement in the cortex. Could the cells of the CAS be using similar mechanisms? We examined sagittal sections for the expression of Nestin, a marker of radial glia. Despite the curvature of the telencephalic ventricle, radial glia projected orthogonal to the ventricle from all along its extent, curving downwards toward the pial surface (Fig. 7a,b). In 

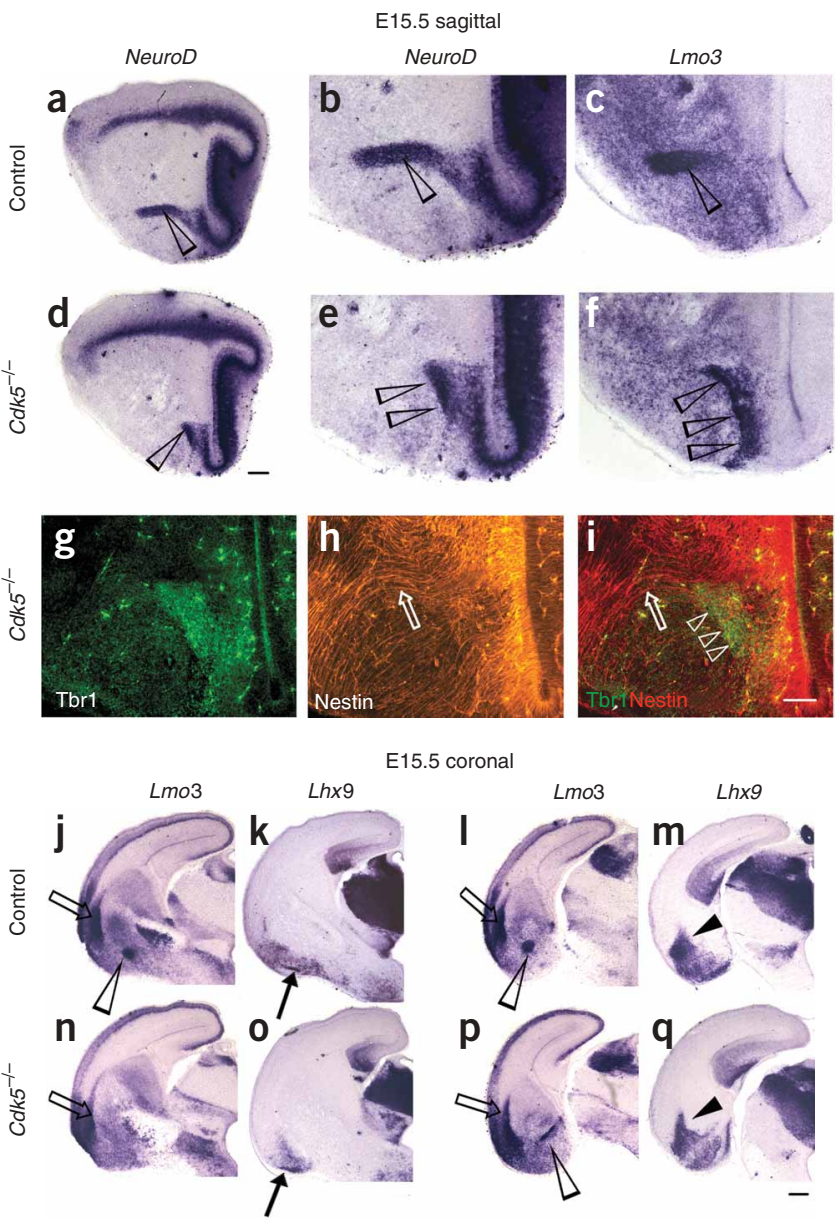

r

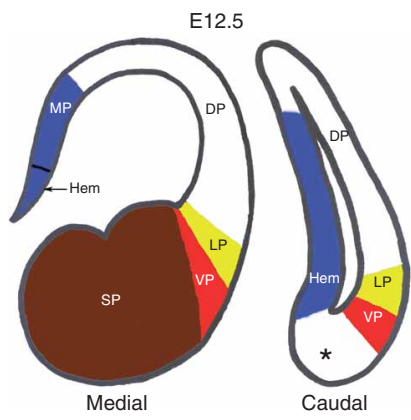

E15.5

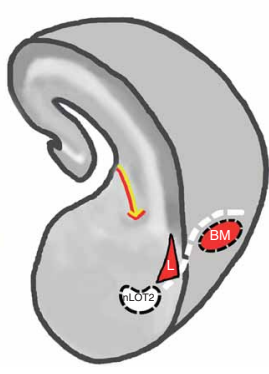

particular, at the caudal pole of the telencephalon, the radial glia extended rostrally for some distance before curving down to touch the ventral pial surface (Fig. 7c). Tbr1 and Nestin immunoreactivity, when examined in the same section, revealed an unexpected complexity in the trajectory of the CAS (Fig. 7d-i). At the origin, and for some distance along its initial trajectory, the CAS appeared to cut across several radial glial fibers, running parallel to the ventricle and orthogonal to the radial glia (arrowheads, Fig. 7d-i). Notably, at a distinct position along its path, the CAS appeared to reorient itself along the direction of the radial glia (arrow, Fig. 7e). This second segment of CAS migration appeared more elongated a day later (arrow, Fig. 7h), at E15.5. This suggests two distinct types of movement at early and late phases of CAS migration.

A key mechanism for migration along radial glia is a pathway that is regulated by Cdk5. This gene is required for migration of the neocorte $\mathrm{x}^{20}$. Specifically, cells in layers $\mathrm{V}$ to II, which utilize radial
Figure 8 Migration of the CAS requires Cdk5. (a-c) NeuroD (a,b) and Lmo3 (c) expression identified the CAS in sagittal sections of control brains at E15.5 (open arrowheads). (d-f) In Cdk5 mutant brains, however, the cells of the CAS appeared to be clustered near the caudal end of the brain (open arrowheads). (g-i) Tbr1 and Nestin double immunoreactivity revealed that the CAS was unable to carry out the second phase of its migration, along the radial glia. The clustering of Tbr1-positive CAS cells was localized in the first segment of the trajectory. Orientation: rostral is left, caudal is right. (j-q) Coronal sections revealed that several other amygdaloid nuclei were unaffected in the Cdk5 mutant. At mid-levels of sectioning, $L$ mo 3 expression marked the $\mathrm{nLOT2}$ (open arrowhead, j) and lateral amygdala (open arrow, j) in control brains. Serial sections showed that the nLOT1 was marked by a thin line of $L h \times 9$ expression (arrow, $\mathbf{k}$ ). In Cdk5 mutant brains, the lateral amygdala (open arrow, $\mathbf{n}$ ) and the nLOT1 (arrow, $\mathbf{0}$ ) were seen, but the $\mathrm{nLOT2}$ appeared to be missing. Sections through caudal levels of control brains showed the $L m o 3$-expressing lateral amygdala (open arrow, I) and nLOT2 (open arrowhead, I) in control brains, as well as the basomedial nucleus marked by $L h \times 9$ expression (arrowhead, $m$ ). In Cdk 5 mutant brains, the lateral amygdala appeared normal (open arrow, $\mathbf{p}$ ), and at this level a deformed line of $L m o 3$ expression was seen (open arrowhead, $\mathbf{p}$ ), which is consistent with the caudally clustered CAS cells seen in $\mathbf{d}-\mathbf{f}$. The basomedial nucleus, expressing $L h \times 9$ (arrowhead, q), appeared normal. Scale bars are $200 \mu \mathrm{m}$. (r) Schematic of an E12.5 brain cut coronally displays the medial, dorsal, lateral and ventral pallial domains (MP, DP, LP, VP, respectively) in the dorsal telencephalon and subpallial domains (SP) in the ventral telencephalon. The hem (the most medial MP structure) is also marked. At caudal levels, the DP appears between the VP and hem (MP) twice - once in its normal, dorsal position and again ventrally (asterisk). The ventral DP corresponds to the source of the CAS. At E15.5, ventrally migrating streams from the neuroepithelium of the LP and VP give rise to different amygdaloid nuclei ${ }^{1,4,6}$. Red and yellow dashed arrows indicate direction of migration from LP and VP, respectively. The CAS migrates orthogonal to the LP/VP migration originating from the caudal telencephalon (dashed white line) and heading rostrally to terminate in the $\mathrm{nLOT2}$. The path of this migration circumnavigates the basomedial nucleus (BM) and terminates ventral to the lateral amygdaloid nucleus (L).

glia-guided migration, fail to reach their correct positions in the absence of Cdk5 function and instead accumulate at incorrect locations beneath the subplate ${ }^{21}$.

We tested the hypothesis that the cells of the CAS migrate using Cdk5-dependent mechanisms. We examined Cdk5 mutant brains at an early stage, E12.5, when amygdaloid migration from the ventral pallium may be detected with specific markers ${ }^{3,4,6}$. Lhx 9 expression, which clearly delineates the ventral pallium stream, appeared normal at several rostro-caudal levels in the absence of Cdk5 (Supplementary Fig. 2 online). In contrast, when $C d k 5$ mutants were probed at E15.5, a profoundly disrupted phenotype was revealed; the migration of the CAS appeared to be arrested close to the ventricular zone, and the cells appear to be piled up at this location, in a vertical line corresponding to the first segment of the CAS trajectory (Fig. 8). Notably, when probed for Tbrl and Nestin immunoreactivity, it was apparent that the CAS cells accumulated along the first stage of their migration (arrowheads, Fig. 8g-i) but did not commence the second stage, which was parallel to the radial glia (arrow, Fig. $\mathbf{8 g}-\mathbf{i}$ ). This suggests that the absence of Cdk5 selectively disrupts a critical radial glial-guided segment of the CAS migration, which is consistent with its established role.

Perhaps the most intriguing feature of the $C d k 5$ mutant amygdala is that the migration defect was specific to the CAS and the nLOT2. Other amygdaloid nuclei were formed at appropriate locations, consistent with the apparently normal ventral pallium stream seen in these mutants. As in the reeler mutant, the nLOT1 forms normally at mid-levels in $C d k 5$ mutant brains. The Lmo3-expressing lateral amygdala/basolateral amygdala and the $L h x 9$-expressing basomedial nucleus also appeared to be 
unaffected in the absence of Cdk5 (Fig. 8). Thus the other components of the pallial amygdala were spared in the Cdk5 mutant, and it was only the CAS and the nLOT2 that were disrupted, once again setting these structures apart from the rest of the amygdaloid complex.

\section{DISCUSSION}

In the telencephalon, distinct regions of the neuroepithelium produce cells that populate specialized structures. Some structures may be located at a distance from their neuroepithelial origins, requiring the cells to undergo complex migration patterns once they are generated. This is particularly true in the case in the amygdaloid complex, whose components arise from distinct, specialized domains in the pallium and the subpallium.

The identification of discrete, molecularly distinct domains near the PSB was an important breakthrough in the fate-mapping of the amygdaloid complex. The ventral pallium in particular was first identified as an intermediate territory in mammalian and nonmammalian species $^{22}$. This led to a series of elegant studies about the structures that arise from this domain, their homologies across species and the transcriptional mechanisms that regulate the ventral pallium domain identity ${ }^{1,3,4}$. Components of the olfactory amygdala are thought to be part of the ventral pallium ${ }^{1,11}$. It is in this context that our study provides new insight. At the extreme caudal end of the telencephalon, the PSB domains are flanked by pallium on both sides, as subpallial structures are not present. The ventral pallium is juxtaposed to the lateral pallium dorsally and was expected to abut the medial pallium ventrally instead of the subpallium (Fig. 8r). Instead, a pallial domain that did not express medial or ventral pallium markers appeared between these two domains at caudal levels. Furthermore, we found that this domain is the origin of components of the amygdaloid complex.

\section{Identity of the neuroepithelial domain that generates the CAS}

Which component of the pallium does the CAS belong to? Four pallial components have been delineated in the telencephalon: the medial, dorsal, lateral and ventral pallia ${ }^{1}$. The ventral pallium has been characterized as a domain that is marked by $D b x 1$ expression at E12.5, but this expression is not maintained at E14.5, which is the earliest stage at which the CAS itself is detectable ${ }^{4}$. However, the origin of the CAS in the ventricular zone can only be assessed at stages when the stream is actually present, making E14.5 the required age for analysis. sFrp2 was characterized as a marker that is maintained in the ventral pallium at E14.5 (ref. 18) and serves as an excellent identifier of the ventral pallium at stages when the CAS is detectable. The ventral pallium has further been characterized to exclude Emxl expression ${ }^{1,3,4}$. The CAS origin domain reveals a completely opposite scenario, with Emxl expression in the ventricular zone and an absence of sFrp2 expression. Furthermore, the nLOT2 appears to be part of the Emx1-expressing lineage, as determined by experiments that marked cells by Emx1-driven Cre-mediated expression of a lacZ reporter $^{23}$. Genetic evidence also indicates that the origin of this structure must lie in Emx1- and Emx2-expressing neuroepithelium, excluding the ventral pallium as a possible source for the nLOT2. Because Emx2 expression covers all pallial domains, including the ventral pallium, whereas Emxl excludes the ventral pallium, the Emx2 (single) mutant mice would have shown a phenotype if the nLOT2 originated from this structure. Furthermore, the ventral pallium is spared in the $L h \times 2$ mutant $^{24}$, and all amygdaloid derivatives of the ventral pallium are present, but the nLOT2 is selectively missing ${ }^{13}$. Finally, the Cdk5 mutant mice show normal ventral pallium migration at E12.5 (Supplementary Fig. 2), which contrasts with the arrested migration of the CAS. Together, these data indicate that the CAS and the nLOT2 arise from a source other than the ventral pallium.

The medial pallium is a domain that includes the hippocampal formation as well as the hem, which was thought to join with the ventral pallium (antihem) in the caudal telencephalon ${ }^{19}$. Because the CAS originates immediately adjacent to the ventral pallium, the hem, which is the most medial part of the medial pallium, is a candidate source for the CAS. The hem is identified by $W n t 2 b$ expression $^{25}$ and excludes $\operatorname{Lh} \times 2$ (ref. 17). In contrast, the CAS origin did not express the hem marker $W n t 2 b$, or any other hem markers (data not shown), and expressed high levels of $L h \times 2$. Furthermore, the $L h \times 2$ mutant has an expanded hem ${ }^{17}$, but the nLOT2 is missing. It is therefore unlikely that the CAS is part of the medial pallium.

Another pallial domain, the lateral pallium, also needs to be assessed as a potential candidate for the origin of the CAS. However, the lateral pallium is located dorsal to the ventral pallium, whereas the CAS originates ventral to the ventral pallium. Furthermore, Cad8, characterized as a lateral pallium marker, is not expressed in the nLOT2, whereas other lateral pallium derivatives such as the claustrum and basolateral nucleus are clearly marked ${ }^{4}$. SCIP, expressed in specific layers of the neocortex and in the nLOT2, does not label lateral pallium derivatives. Thus, the lateral pallium is not a likely source for the CAS.

An examination of the E12.5 telencephalon revealed how the dorsal pallium extends itself over the caudal pole of the telencephalon, and appears to be protruding between the ventral and medial pallium, in the location corresponding to the origin of the CAS (Fig. $4 \mathbf{v}, \mathbf{w}$ ). The broad array of pallial markers that are expressed in the dorsal pallium, NeuroD, NeuroD2, Math2, Tbr1 and Emx1, are also expressed either in the CAS origin in the ventricular zone or in the migrating cells of the CAS and in the nLOT2, indicating that these structures are components of the dorsal pallium. This is the first evidence of a dorsal pallium contribution to the mammalian amygdaloid complex.

\section{Is the nLOT a single trilaminar nucleus?}

The nLOT is described as a trilaminar nucleus, and like other cortical nuclei, it is considered to be an extension of the trilaminar piriform cortex $^{8,11,26}$. A previous study ${ }^{4}$ reported distinct marker expression of the nLOT2 compared with nLOT1 and 3, suggesting that there is an intermingling of cells from distinct origins in the different layers of the nLOT. Consistent with this analysis, we found fundamental developmental differences between the nLOT1 and 2, indicating that these may in fact be independent structures instead of components of a single laminar unit. These data are relevant to the current debate that questions the identity of the entire amygdaloid complex as a unit, and propose instead four different functional subgroups ${ }^{8,26-28}$. Our study adds further complexity to such groupings, such that the nLOT itself is not a layered unit but a collection of independently formed nuclei, of which the nLOT2 represents a developmental connection with the neocortex.

\section{Reelin- and Cdk5-dependent migration of the CAS}

Normal migration of the CAS/nLOT2 requires reelin and Cdk5, which is consistent with a dorsal pallium affiliation, as the neocortex also requires these mechanisms for its migration ${ }^{20,29}$. Reelin is an extracellular signal, whereas Cdk5 is an intracellular kinase, yet mutations in these genes have partially similar phenotypes in the neocortex ${ }^{20,29}$. These two pathways function in parallel and are thought to intersect at the level of downstream effectors, including those that regulate cytoskeletal dynamics in the cell ${ }^{30,31}$.

Cdk5 mutants have disrupted migration specifically of the late-born layers of the cortex, but not of the cells of the preplate or layer $\mathrm{VI}^{21}$. This is thought to be due to different modes of migration used by early-born 
versus late-born cells ${ }^{32}$, possibly as a result of differences in the distances they need to travel. Cells of the upper layers need to travel longer distances and use radial glial-guided mechanisms to reach their final locations $s^{33,34}$. Though the cells of the CAS are born at stages corresponding to the early-born deep layers of the cortex, they have to migrate much farther from their site of origin. They appear to initially utilize Cdk5-independent mechanisms but later on require Cdk5dependent mechanisms, similar to the superficial layers of the cortex. Other amygdaloid nuclei, however, do not appear to require Cdk5 to achieve their migration, even though radial glia extend from the ventricular zone to all these structures. Only the migration of the CAS is markedly arrested in the absence of Cdk5, similar to the phenotype in the superficial layers of the cortex.

In contrast, in reeler mice, the cells of the CAS and the nLOT2 appear to be affected in a somewhat different manner from the lamination defects reported for the cortex. Whereas the cortical layers become 'inverted' in the absence of reelin ${ }^{29}$, the CAS and the nLOT2 cells appear to be immature and are trapped in a route that is shorter and misdirected. What might these differences suggest? It is significant that $D a b-1$, a key mediator of reelin signaling in the cortex ${ }^{35}$, is expressed strongly in the cortex and in the nLOT2 but is not detectable in many other pallial amygdaloid components, such as the lateral amygdala, basolateral amygdala and basomedial nucleus. This suggests that except for the nLOT2 and the anterior amygdala, which express Dab-1, other amygdaloid nuclei would not be able to process the reelin signal via known mechanisms, in which Dab-1 is a necessary and critical component ${ }^{35}$. Consistent with this interpretation, the lateral amygdala, basolateral amygdala and basomedial nucleus, derived from the ventral and lateral pallia, appear to be unaffected in the reeler mutant.

We have shown that the nLOT2 is developmentally linked to the neocortex by specific markers, by shared mechanisms of specification (Lhx2, Tbr1 and Emx1/2), by Dab-1 expression and by Cdk5-dependent mechanisms of migration. The additional commonality of reelindependent mechanisms for migration offers useful evolutionary insights. Amygdaloid structures or their homologs have been identified in reptiles and birds, as well as mammals, and predate the appearance of the neocortex, which is unique to mammals ${ }^{1,11,36-42}$. Understanding how laminated neocortex arose is a difficult and open question, as common ancestors between mammals and nonmammalian organisms are extinct, and there is no information on how the transition to a layered neocortical organization was achieved ${ }^{43-45}$. One hypothesis is based on the observation that the reelin signal is substantially amplified in mammals compared with other vertebrates, suggesting that an acquired dependence on reelin-guided mechanisms for regulating cell migration may have led to the formation of layered neocorte ${ }^{44,45}$. It is tempting to speculate that, because the nLOT is present in reptiles ${ }^{38}$, this nucleus may have been among the first structures to recruit the reelin pathway for its migration, in a manner that may have preceded the role of reelin in the formation of the neocortex. Thus the nLOT2, a unique a structure from the present-day mammalian brain, reveals a shared developmental history with the neocortex. This finding may offer an insight into the evolutionary transition from a clustered (nuclear) organization of cells to the highly laminated arrangement that is the defining feature of the neocortex.

\section{METHODS}

Animals. All procedures followed the Institute Animal Ethics Committee guidelines and US National Institutes of Health guidelines for the care and use of animals. The Tata Institute Instutional Animal Ethics Committe approved the experiments. Timed-pregnant Swiss mice were obtained from the Tata Institute animal breeding facility. Tbr1, Emx1, Emx1/2, Reeler, Cdk5 and Lhx2 mutant embryos were obtained by intercrossing mice that were heterozygous for the respective mutant alleles ${ }^{15,16,20,46-48}$.

In situ hybridization. Hybridization was carried out as described previously ${ }^{17}$. Details are in the Supplementary Methods online.

Immunohistochemistry. BrdU labeling and detection were carried out as previously described ${ }^{49}$, except that the secondary antibody used for fluorescence detection was biotinylated, followed by detection with streptavidin conjugated to Alexa Fluor 488 (Molecular Probes). Tbr1 antibody ${ }^{15}$ and Nestin immunohistochemistry (using monoclonal antibody Rat-401 supernatant (Developmental Studies Hybridoma Bank, 1:10 dilution)) were carried out using standard antigen retrieval protocols, as described in the Supplementary Methods.

Imaging. Images were grabbed using an Axiocam via Axiovision software (Carl Zeiss). False color overlays were made as described previously ${ }^{3}$.

In utero electroporation. $2.5 \%$ avertin (2,2,2-tribromoethanol, $97 \%$ in tertamylalcohol, Aldrich) in $0.9 \%$ saline was injected intraperitonially ( $15 \mu \mathrm{l}$ per g of body weight) to anaesthetize timed-pregnant mice at E11.5-12.5. Electroporation was carried out as described previously ${ }^{50}$ and is described in detail in the Supplementary Methods.

Note: Supplementary information is available on the Nature Neuroscience website.

\section{ACKNOWLEDGMENTS}

We thank R. Kageyama, Y. Nakagawa, F. D. Porter, T. Rabbitts, C. Ragsdale, E. Grove and Y. Zhao for gifts of plasmid DNA; F. D. Porter for the Lhx2 mutant breeding pairs; $M$. Frotscher for the gift of reeler mutant tissue for preliminary experiments; and V. Vaidya, M. Benekareddy and A. Nair for critical comments on the manuscript. The Rat-401 monoclonal antibody to Nestin, developed by S. Hockfield, was obtained from the Developmental Studies Hybridoma Bank and was developed under the auspices of the US National Institute of Child Health and Human Development and maintained by The University of Iowa. We also thank the Tata Institute of Fundamental Research Animal House staff for excellent support. This work was supported by a Wellcome Trust Senior Fellowship (056684/ Z/99/Z) and a Swarnajayanti Fellowship (Dept. of Science and Technology, Govt. of India) to S.T. and a Kanwal Rekhi Career Development Award (Tata Institute of Fundamental Research Endowment Fund) to B.S. and P.H.

\section{AUTHOR CONTRIBUTIONS}

R.R., D.H., B.S., P.H. and L.B. carried out the experiments. R.R., D.H. and S.T. analyzed the data. S.T. supervised the project and wrote the paper. Mutant mouse tissue was provided by T.K. and R.F.H. (Tbrl mutant and preliminary data), Y.S. and S.A. (Emx1 and Emx1/2 double mutants), T.O. (Cdk5 mutant) and A.S. (Emx2 and reeler mutants).

\section{COMPETING INTERESTS STATEMENT}

The authors declare no competing financial interests.

Published online at http://www.nature.com/natureneuroscience Reprints and permissions information is available online at http://npg.nature.com/ reprintsandpermissions

1. Puelles, L. et al. Pallial and subpallial derivatives in the embryonic chick and mouse telencephalon, traced by the expression of the genes Dlx-2, Emx-1, Nkx-2.1, Pax-6 and Tbr-1. J. Comp. Neurol. 424, 409-438 (2000).

2. Nery, S., Fishell, G. \& Corbin, J.G. The caudal ganglionic eminence is a source of distinct cortical and subcortical cell populations. Nat. Neurosci. 5, 1279-1287 (2002).

3. Yun, K., Potter, S. \& Rubenstein, J.L. Gsh2 and Pax6 play complementary roles in dorsoventral patterning of the mammalian telencephalon. Development 128, 193-205 (2001).

4. Medina, L. et al. Expression of Dbx1, Neurogenin 2, Semaphorin 5A, Cadherin 8 and Emx1 distinguish ventral and lateral pallial histogenetic divisions in the developing mouse claustroamygdaloid complex. J. Comp. Neurol. 474, 504-523 (2004).

5. Stenman, J., Yu, R.T., Evans, R.M. \& Campbell, K. TIx and Pax6 cooperate genetically to establish the pallio-subpallial boundary in the embryonic mouse telencephalon. Development 130, 1113-1122 (2003).

6. Tole, S., Remedios, R., Saha, B. \& Stoykova, A. Selective requirement of Pax6, but not Emx2, in the specification and development of several nuclei of the amygdaloid complex. J. Neurosci. 25, 2753-2760 (2005).

7. Alheid, G.F., de Olmos, J. \& Beltramino, C.A. Amygdala and extended amygdala in the rat nervous system. in The Rat Nervous System, $2^{\text {nd }}$ ed. (ed. Gaxinos, P.) 495-578 (Academic Press, San Diego, California, 1995). 
8. McDonald, A.J. Is there an amygdala and how far does it extend? An anatomical perspective. Ann. NY Acad. Sci. 985, 1-21 (2003).

9. Price, J.L. An autoradiographic study of complementary laminar pattern of termination of afferent fibres to the olfactory cortex. J. Comp. Neurol. 150, 87-108 (1973).

10. Luskin, M.B. \& Price, J.L. The topographic organization of associational fibres of the olfactory system in the rat, including centrifugal fibers to the olfactory bulb. J. Comp. Neurol. 216, 264-291 (1983).

11. Martinez-Garcia, F., Martinez-Marcos, A. \& Lanuza, E. The pallial amygdala of amniote vertebrates: evolution of the concept, evolution of the structure. Brain Res. Bull. 57, 463-469 (2002).

12. Santiago, A.C. \& Shammah-Lagnado, S.J. Efferent connections of the nucleus of the lateral olfactory tract in the rat. J. Comp. Neurol. 471, 314-332 (2004).

13. Remedios, R., Subramanian, L. \& Tole, S. LIM genes parcellate the embryonic amygdala and regulate its development. J. Neurosci. 24, 6986-6990 (2004).

14. McConnell, J. \& Angevine, J.B., Jr. Time of origin in the amygdaloid complex of the mouse. Brain Res. 272, 150-156 (1983).

15. Hevner, R.F. et al. Tbr1 regulates differentiation of the preplate and layer 6. Neuron 29, 353-366 (2001).

16. Shinozaki, K. et al. Absence of Cajal-Retzius cells and subplate neurons associated with defects of tangential cell migration from ganglionic eminence in Emx1/2 double mutant cerebral cortex. Development 129, 3479-3492 (2002).

17. Bulchand, S., Grove, E.A., Porter, F.D. \& Tole, S. LIM-homeodomain gene Lhx2 regulates the formation of the cortical hem. Mech. Dev. 100, 165-175 (2001).

18. Kim, A.S., Anderson, S.A., Rubenstein, J.L., Lowenstein, D.H. \& Pleasure, S.J. Pax-6 regulates expression of SFRP-2 and Wnt-7b in the developing CNS. J. Neurosci. 21, RC132 (2001).

19. Assimacopoulos, S., Grove, E.A. \& Ragsdale, C.W. Identification of a Pax6-dependent epidermal growth factor family signaling source at the lateral edge of the embryonic cerebral cortex. J. Neurosci. 23, 6399-6403 (2003).

20. Ohshima, T. et al. Targeted disruption of the cyclin-dependent kinase 5 gene results in abnormal corticogenesis, neuronal pathology and perinatal death. Proc. Natl. Acad. Sci. USA 93, 11173-11178 (1996).

21. Gilmore, E.C., Ohshima, T., Goffinet, A.M., Kulkarni, A.B. \& Herrup, K. Cyclindependent kinase 5-deficient mice demonstrate novel developmental arrest in cerebral cortex. J. Neurosci. 18, 6370-6377 (1998).

22. Fernandez, A.S., Pieau, C., Reperant, J., Boncinelli, E. \& Wassef, M. Expression of the Emx1 and Dlx-1 homeobox genes define three molecularly distinct domains in the telencephalon of mouse, chick, turtle and frog embryos: implications for the evolution of telencephalic subdivisions in amniotes. Development 125, 2099-2111 (1998).

23. Gorski, J.A. et al. Cortical excitatory neurons and glia, but not GABAergic neurons, are produced in the Emx1-expressing lineage. J. Neurosci. 22, 6309-6314 (2002).

24. Vyas, A., Saha, B., Lai, E. \& Tole, S. Paleocortex is specified in mice in which dorsal telencephalic patterning is severely disrupted. J. Comp. Neurol. 466, 545-553 (2003).

25. Grove, E.A., Tole, S., Limon, J., Yip, L. \& Ragsdale, C.W. The hem of the embryonic cerebral cortex is defined by the expression of multiple Wnt genes and is compromised in Gli3-deficient mice. Development 125, 2315-2325 (1998).

26. Swanson, L.W. \& Petrovich, G.D. What is the amygdala? Trends Neurosci. 21, 323-331 (1998).

27. Barton, R.A., Aggleton, J.P. \& Grenyer, R. Evolutionary coherence of the mammalian amygdala. Proc Biol Sci. 270, 539-543 (2003).

28. Swanson, L.W. The amygdala and its place in the cerebral hemisphere. Ann. NY Acad. Sci. 985, 174-184 (2003).
29. Caviness, V.S., Jr. Neocortical histogenesis in normal and reeler mice: a developmental study based upon [3H]thymidine autoradiography. Brain Res. 256, 293-302 (1982).

30. Beffert, U. et al. Reelin and cyclin-dependent kinase 5-dependent signals cooperate in regulating neuronal migration and synaptic transmission. J. Neurosci. 24, 1897-1906 (2004).

31. Xie, Z., Samuels, B.A. \& Tsai, L.H. Cyclin-dependent kinase 5 permits efficient cytoskeletal remodeling-a hypothesis on neuronal migration. Cereb. Cortex 16, i64-i68 (2006).

32. Nadarajah, B., Brunstrom, J.E., Grutzendler, J., Wong, R.O. \& Pearlman, A.L. Two modes of radial migration in early development of the cerebral cortex. Nat. Neurosci. 4 , 143-150 (2001).

33. Nadarajah, B. \& Parnavelas, J.G. Modes of neuronal migration in the developing cerebral cortex. Nat. Rev. Neurosci. 3, 423-432 (2002).

34. Hatanaka, Y., Hisanaga, S., Heizmann, C.W. \& Murakami, F. Distinct migratory behavior of early and late-born neurons derived from the cortical ventricular zone. J. Comp. Neurol. 479, 1-14 (2004).

35. Rice, D.S. et al. Disabled-1 acts downstream of Reelin in a signaling pathway that controls laminar organization in the mammalian brain. Development 125, 3719-3729 (1998).

36. Streidter, G.F. The telencephalon of tetrapods in evolution. Brain Behav. Evol. 49, 179-213 (1997).

37. Aboitiz, F., Montiel, J., Morales, D. \& Concha, M. Evolutionary divergence of the reptilian and the mammalian brains: considerations on connectivity and development. Brain Res Brain Res Rev. 39, 141-153 (2002).

38. Martinez-Garcia, F., Olucha, F.E., Teruel, V., Lorente, M.J. \& Schwerdtfeger, W.K. Afferent and efferent connections of the olfactory bulbs in the lizard Podarcis hispanica. J. Comp. Neurol. 305, 337-347 (1991).

39. Molnar, Z. \& Butler, A.B. The corticostriatal junction: a crucial region for forebrain development and evolution. Bioessays 24, 530-541 (2002).

40. Yamamoto, K., Sun, Z., Wang, H.B. \& Reiner, A. Subpallial amygdala and nucleus taeniae in birds resemble extended amygdala and medial amygdala in mammals in their expression of markers of regional identity. Brain Res. Bull. 66, 341-347 (2005).

41. Aboitiz, F. Evolution of isocortical organization. A tentative scenario including roles of reelin, p35/cdk5 and the subplate zone. Cereb. Cortex 9, 655-661 (1999).

42. Broccoli, V.V. Evolutionary developmental biology of the cerebral cortex. Bioessays 21 , 974-977 (1999).

43. Avian Brain Nomenclature Consortium. Avian brains and a new understanding of vertebrate brain evolution. Nat. Rev. Neurosci. 6, 151-159 (2005).

44. Bar, I., Lambert de Rouvroit, C. \& Goffinet, A.M. The evolution of cortical development A hypothesis based on the role of the Reelin signaling pathway. Trends Neurosci. 23, 633-638 (2000).

45. Tissir, F., Lambert de Rouvroit, C. \& Goffinet, A.M. The role of Reelin in the development and evolution of the cerebral cortex. Braz. J. Med. Biol. Res. 35, 1473-1484 (2002).

46. D'Arcangelo, G. et al. A protein related to extracellular matrix proteins deleted in the mouse mutant reeler. Nature 374, 719-723 (1995).

47. Pellegrini, M., Mansouri, A., Simeone, A., Boncinelli, E. \& Gruss, P. Dentate gyrus formation requires Emx2. Development 122, 3893-3898 (1996).

48. Porter, F.D. et al. Lhx2, a LIM homeobox gene, is required for eye, forebrain and definitive erythrocyte development. Development 124, 2935-2944 (1997).

49. Tole, S. \& Grove, E.A. Detailed field pattern is intrinsic to the embryonic mouse hippocampus early in neurogenesis. J. Neurosci. 21, 1580-1589 (2001).

50. Saito, T. \& Nakatsuji, N. Efficient gene transfer into the embryonic mouse brain using in vivo electroporation. Dev. Biol. 240, 237-246 (2001). 